\title{
La « pensée ingénieuse » dans les pièces de Racine
}

\author{
Jean-Yves Vialleton
}

\section{(2) OpenEdition}

Journals

Édition électronique

URL : http://journals.openedition.org/rhetorique/93

DOI : $10.4000 /$ rhetorique.93

ISSN : 2270-6909

\section{Éditeur}

UGA Éditions/Université Grenoble Alpes

\section{Édition imprimée}

ISBN : 978-2-84310-255-4

\section{Référence électronique}

Jean-Yves Vialleton, «La " pensée ingénieuse » dans les pièces de Racine », Exercices de rhétorique [En ligne], 1 | 2013, mis en ligne le 06 novembre 2013, consulté le 12 septembre 2020. URL : http:// journals.openedition.org/rhetorique/93; DOI : https://doi.org/10.4000/rhetorique.93

Ce document a été généré automatiquement le 12 septembre 2020.

\section{(c) (i) (2)(2)}

Les contenus de la revue Exercices de rhétorique sont mis à disposition selon les termes de la Licence Creative Commons Attribution - Pas d'Utilisation Commerciale - Partage dans les Mêmes Conditions 4.0 International. 


\title{
La « pensée ingénieuse » dans les pièces de Racine
}

\author{
Jean-Yves Vialleton
}

On a souvent remarqué que la première pièce conservée de Racine, La Thébaïde, était remplie de "pointes", pointes que les corrections faites au cours des éditions successives n'ont pas effacées. C'est sur cette observation qu'on voudrait revenir et on le fera en prenant pour guide le Révérend Père Bouhours en tant que théoricien de la " pensée ingénieuse ${ }^{1}$ ».

Dominique Bouhours ${ }^{2}$ (1628-1702 : Jean Racine est né en 1639) est un professeur jésuite $\mathrm{du}$ collège de Clermont, auteur de livres religieux, d'édification ou de polémique. Mais c'est aussi un bel esprit présent au samedi de Mlle de Scudéry et une autorité en matière de bon usage du français, un " remarqueur » si juste qu'il est considéré dans les années 1670 comme un nouveau Vaugelas. Par une de ses lettres, Racine lui soumet les quatre premiers actes de la tragédie qu'il est en train d'écrire ${ }^{3}$. Bouhours est enfin dans les années 1680 , et c'est ce qui nous intéresse ici, un théoricien de la "pensée ingénieuse ». C'est le sujet de son livre intitulé La Manière de bien penser dans les ouvrages de l'esprit. Dialogue (1687), ouvrage à succès (il connaît de très nombreuses rééditions ${ }^{4}$ ). Dans l'« Avertissement ${ }^{5}$ », Bouhours présente son ouvrage comme "une Logique sans épines" et une "Rhétorique courte et facile ", même s'il prévient qu'il ne prétend "rien enseigner de l'Art oratoire». Le livre est composé de quatre dialogues entre Eudoxe et Philanthe (en grec, la bonne doctrine et l'amateur de fleurs), celui-ci étant un admirateur duTasse et de Lope de Vega, celui-là prônant une esthétique plus raisonnable. Par sa forme même, celle du dialogue familier, le livre se présente comme une suite des Entretiens d'Ariste et d'Eugène (1671), best-seller ${ }^{6}$ auquel l'« Avertissement " renvoie explicitement, le livre de Bouhours aujourd'hui le plus connu' ${ }^{7}$. La Manière de bien penser a à son tour une suite, les Pensées ingénieuses des anciens et des modernes $\left(1689^{8}\right)$, qui abandonne la forme du dialogue et se présente comme une simple anthologie commentée. Dans La Manière de bien penser, Eudoxe s'appuyait sur divers livres et surtout sur un recueil de passages d'auteurs qu'il s'était composé et dont il disait extraire les nombreuses citations qui illustraient sa réflexion. De ce « recueil de 
lieux communs ${ }^{9}$ ", les Pensées ingénieuses de 1689 offrent au lecteur toutes les citations qui n'ont pas été utilisées en 1687 dans La Manière de bien penser.

Bouhours est cité comme un théoricien du classicisme («Au panier les Bouhours!» s'est écrié Hugo). Il l'est évidemment en ce qu'il prône la raison, le «bon sens », et qu'il blâme ce que Boileau appelle le "clinquant" des Italiens du XvI siècle. Cependant Bouhours ne prône pas exactement le « bon sens », mais le «bon sens vif et brillant ${ }^{10}$ »: mettre l'accent sur le judicium (la culture, le sens critique), n'est pas renier l'ingenium (la créativité, le don). Bouhours s'oppose certes explicitement aux conceptistes italiens et espagnols qui théorisent les vivezze d'ingenio, les agudezas, en particulier Gracián et Tesauro, dont le Connochiale Aristotelico est cité ${ }^{11}$. Mais c'est justement parce qu'il se situe dans le même champ qu'eux, la "rhétorique de la pointe", pour reprendre le titre de l'étude de Mercedes Blanco ${ }^{12}$. Il choisit la forme mondaine du dialogue et non celle du lourd traité « aristotélique » des conceptistes, mais il utilise au fond la même méthode qu'eux (recueillir des morceaux choisis, les mettre en série, les classer, les évaluer), méthode qui fait de ces ouvrages des sortes d'anthologies commentées, aspect qu'ont bien des ouvrages de rhétorique antiques et modernes: le Parallèle de l'éloquence profane et sacrée du Père Caussin, fameux depuis les travaux de Marc Fumaroli, peut être lu comme un ouvrage théorique, mais aussi comme une anthologie commentée. Comme les conceptistes, Bouhours range dans la pensée ingénieuse la "facétie " aussi bien que le mot sublime. On sent une inclination de Gracián pour les mots sublimes et au contraire chez Bouhours un goût pour les «pensées ingénieuses » de Voiture, c'est-à-dire celles du style simple et piquant. Mais Bouhours comme les conceptistes considère la pensée ingénieuse dans ses différents registres rhétoriques ${ }^{13}$ : elle peut être dans le " genre noble ", " agréable », « délicat ", c'est-à-dire relever de chacun des «styles » rhétoriques. Enfin, Bouhours s'accorde avec les conceptistes pour considérer, comme une "pensée » selon ses propres mots, comme un " acte de l'entendement » selon ceux de Gracián ${ }^{14}$, ce que nous ne considérons plus que sous l'angle de la figure d'élocution. On peut orner son discours par une métaphore ou une antithèse. Mais mettre en évidence une analogie entre deux choses ou éclairer une chose en la rapprochant de son contraire sont avant tout des mécanismes de la créativité, dans la terminologie rhétorique des «lieux» de l'«invention ${ }^{15}$ ». La "pensée » est "ingénieuse » en ce qu'elle relève non de l'élocution, mais plus profondément de l'invention, et donc de l'ingenium, d'une difficile logique de la trouvaille. Aussi la pensée ingénieuse peut-elle se limiter à une phrase, mais aussi bien fonder toute une œuvre.

\section{D'une pensée ingénieuse à propos du visage d'un mort}

Le récit de la mort d'Étéocle et de Polynice dans la Thébaïde de Racine s'achève par ces vers :

Il [Étéocle] lui perce le cœur ; et son âme ravie,

En achevant ce coup, abandonne la vie.

Polynice frappé pousse un cri dans les airs,

Et son âme en courroux s'enfuit dans les Enfers.

Tout mort qu'il est, Madame, [Créon s'adresse à Antigone] il garde sa colère ;

Et l'on dirait qu'encore il menace son frère.

Son visage, où la mort a répandu ses traits, 
Demeure plus terrible et plus fier que jamais.

[Thébaïde, V, 3, en 1664, v. 1517-1524 ; en 1697, v. 1377-1384]

5 Le vers « Et son âme en courroux [...] » est, comme le rappelle une note des éditions par G. Forestier ${ }^{16}$, une imitation du tout dernier vers de l'Énéide (chant XII, v. 950), évoquant la mort de Turnus :

hoc dicens ferrum aduerso sub pectore condit

feruidus. Ast illi soluuntur frigore membra

uitaque cum gemitu fugit indignata sub umbras.

«Ce disant, il lui plonge son fer en pleine poitrine, tout bouillant.

Les membres de l'autre se détendent sous le froid de la mort,

et sa vie indignée s'enfuit, avec un gémissement, chez les ombres ${ }^{17}$. »

Le dernier vers de l'Énéide est lui-même une imitation, celle d'un passage de l'Iliade sur la mort d'Hector (chant XXII, v. 362) et il fonde un motif dont les romanzi et les poèmes héroïques italiens donnent plusieurs variations. L'Orlando furioso de l'Arioste finit par la mort de Rodomont tué par Roger, comme l'Énéide par celle de Turnus :

Alle squalide ripe d'Acheronte,

sciolta dal corpo più freddo che giaccio,

bestemmiando fuggì l'alma sdegnosa,

che fu sì altiera al mondo e sì orgogliosa.

«Vers les affreuses rives d'Achéron,

délivrée du corps plus froid que glace,

s'enfuit, en blasphémant, l'âme dédaigneuse

qui fut si altière et si orgueilleuse au monde ${ }^{18}$. »

Mais Racine ajoute quatre vers qui décrivent le visage de Polynice mort, formulant trois pointes successives et chaque fois plus fortes. Racine nous montre quelque chose d'à peine croyable, où la réalité semble irréelle, un thaumaston, une maraviglia, le prodige d'un homme à la fois déjà mort et encore vivant. C'est sur le même motif, employé également comme point d'orgue saisissant, que le cinéaste Claude Lanzmann clôt l'exorde, je veux dire le premier chapitre, de son autobiographie récente. Il s'agit ici non d'une description, mais, dans la tradition de la seconde sophistique (Philostrate) et aussi par un procédé cher au roman français contemporain, de la description d'une image (une image arrêtée de la vidéo d'une exécution) :

Le visage de l'égorgé et celui du vivant qu'il était se ressemble irréellement. C'est le même visage et c'est à peine croyable : la sauvagerie de cette mise à mort était telle qu'elle semblait ne pouvoir se sceller que d'une radicale défiguration ${ }^{19}$.

Racine développe peut-être une indication de Virgile (la vie de Turnus s'en va, mais elle est indignée), mais en l'exagérant jusqu'à l'impossible (tout mort qu'il est, il garde sa colère) et en employant le registre stylistique que la rhétorique hellénistique croyait devoir distinguer de la grandeur, le style deinos, véhément, la terribilità. En témoigne par exemple l'allitération en $r$ des deux derniers vers. Il reprend surtout Stace, mais en allant plus loin. Dans le livre XI de sa propre Thébaïde, le jeu sur la mort et la vie est présent, mais Stace reste dans la vraisemblance. Polynice, après avoir reçu le coup de son frère qu'il croyait mort, lui dit :

Ille autem : "Vivisne an adhuc manet ira superstes

perfide, nec sedes unquam meriture quietas?"

"Mais celui-ci : "Es-tu vivant ou bien la colère te survit-elle encore

perfide, qui jamais ne doit mériter le séjour de la paix ${ }^{20}$ ?" "

Faisant cela, Racine a pu se souvenir de la manière dont Corneille dans Pompée (1644) s'y était pris pour décrire la tête décapitée de Pompée présentée à César, scène imitée dans 
d'autres tragédies avec décapitation. Corneille se déclare imitateur de Lucain jusqu'à donner la référence des vers paraphrasés, mais dans cette description, il reprend Lucain en le dépassant en audace. Dans La Pharsale, Lucain décrit le visage de Pompée au moment de sa mort, au livre VIII (v. 665 sq.) :

At, Magni cum terga sonent et pectora ferro,

Permansisse decus sacrae uenerabile formae

Iratamque deis faciem, nil ultima mortis

Ex habitu uoltuque uiri mutasse fatentur

« Pompée en expirant

avait conservé sur son visage vénérable l'empreinte de la majesté.

On n'y voyait que de l'indignation contre les dieux,

l'effort même de l'agonie n'avait point altéré ses traits. »

Dans sa traduction, Brébeuf est fidèle, mais ajoute une pointe de terribilità :

Pompée au même instant sous l'effet de la rage

Achève son trépas sans changer de visage,

Son calme semble encore y vivre après sa mort,

Un mépris généreux y brave un dur effort,

Et son âme insensible à sa propre souffrance,

semble avoir laissé toute son assurance.

[...]

Et cet illustre mort donne encore de la crainte $\mathrm{e}^{21}$.

11 Au livre IX de La Pharsale, la tête de Pompée est coupée, momifiée, puis apportée à César, qui, en la voyant, d'abord ne la reconnaît pas. Corneille s'inspire du premier passage pour le second :

À ces mots Achillas découvre cette tête :

Il semble qu'à parler encore elle s'apprête.

Qu'à ce nouvel affront un reste de chaleur

En sanglots mal formés exhale sa douleur ;

Sa bouche encore ouverte et sa vue égarée

Rappellent sa grande âme à peine séparée,

Et son courroux mourant fait un dernier effort

Pour reprocher aux Dieux sa défaite et sa mort.

César, à cet aspect, comme frappé du foudre ${ }^{22}[\ldots]$.

12 Le Père Bouhours a justement souvent cité et commenté, dans ses différents livres, des «pensées ingénieuses » construites sur le motif du tué qui est encore vivant. C'est sur ce motif qu'est composée la devise citée dans les Entretiens d'Ariste et d'Eugène qui a pour " corps" un serpent coupé en deux et pour "âme » nec mors, nec vita relictae ${ }^{23}$. Dès le premier dialogue de La Manière de bien penser, Bouhours critique une citation célèbre fondée sur ce même motif :

De l'humeur dont vous êtes, répliqua Philanthe, vous n'approuveriez pas ce que dit l'Arioste d'un de ses Héros; que dans la chaleur du combat, ne s'étant pas aperçu qu'on l'avait tué, il combattit toujours vaillamment tout mort qu'il était.

Il pover' huomo che non s'en era accorto

Andava combattendo, ed era morto ${ }^{24}$.

"Le pauvre homme qui ne s'en était pas aperçu

Allait combattant, et il était mort. »

13 Cette citation était célèbre au XVII ${ }^{e}$ siècle: on la trouve chez Mme de Sévigné25 (spirituellement « appliquée » à un homme dont elle apprend la mort et qu'elle revoit encore il y a peu jouer au piquet). Le passage de Bouhours lui permet de le rester : elle devient la citation presque obligée en France quand on parle de l'Arioste, et l' 
Encyclopédie de Diderot à l'article "Arioste" ne manque pas de la donner. Cela est savoureux, car la citation n'est pas tirée de l'Arioste, mais d'une épopée burlesque, une parodie de Boiardo, l'Orlando innamorato de Berni (le Sarrasin Alaband de Tolède, coupé en deux, continue à se battre). La critique moderne souvent l'ignore, mais cela n'a pas échappé aux auteurs du Menagiana, et la recherche électronique automatique permettra d'éviter de retomber dans le piège. Le premier vers de la citation est d'ailleurs inexact et faux métriquement, il sert à accentuer le burlesque. Le bon texte est :

Cosi colui, del colpo non accorto

Andava combattendo, ed era morto ${ }^{26}$.

Bouhours enchaîne avec d'autres citations :

Je n'approuve pas même, repartit Eudoxe, ce que le Tasse dit d'Argant:

Minacciava morendo, e non languia.

Je vous abandonne l'Arioste, reprit Philanthe ; mais je vous demande quartier pour le Tasse, et je vous prie de considérer qu'un Sarasin robuste et féroce qui a été blessé dans le combat et qui meurt de ses blessures, peut bien menacer en mourant celui qui lui donne le coup de la mort. Je consens qu'il le menace, répondit Eudoxe, et même que ses derniers gestes, que ses dernières paroles, aient quelque chose de fier, de superbe, et de terrible.

Superbi, formidabili, feroci

Gli ultimi moti fur, l'ultime voci.

Cela peut être, et cela convient au caractère d'Argant : à la mort on conserve les sentiments qu'on a eus pendant la vie ; on ramasse ce qui reste d'esprits et de forces pour exprimer ce qu'on sent; on jette quelquefois des cris effroyables avant que de rendre le dernier soupir: mais de n'être point faible lorsqu'on se meurt, $e$ non languia, c'est ce qui n'a point de vraisemblance. Le Cannibale de Montaigne est bien plus dans la nature que le Sarasin du Tasse. Car enfin si le Cannibale prisonnier de ses ennemis les brave jusques dans les fers, leur dit des injures, leur crache au visage ; si au milieu des tourments et sur le point de mourir, n'ayant pas la force de parler, il leur fait la moue pour se moquer d'eux, et pour leur témoigner qu'il n'est pas vaincu : il n'y a rien là qui ne soit conforme au génie d'un barbare fier et tout plein de cœur.

Mais qu'y a-t-il de plus convenable à la vertu héroïque, dit Philanthe, que de mourir sans nulle faiblesse? Les Héros, reprit Eudoxe, ont de la constance en mourant; mais la fermeté de leur âme n'empêche pas que leur corps ne s'affaiblisse ; ils n'ont de ce côté-là nul privilège. Cependant le non languia qui va au corps, exempte Argant de la loi commune, et détruit l'homme en élevant le Héros.

Je crains, repartit Philanthe, que votre délicatesse n'aille trop loin, et que vous n'outriez un peu la Critique. Le Tasse veut dire, ce me semble, qu'à voir Argant irrité contre Tancrède, et le menaçant sur le point de mourir, on n'eût pas dit qu'il se mourait; que sa fierté et sa colère effaçaient en quelque sorte sa langueur, et le faisaient paraître vigoureux.

C'est dommage, répliqua Eudoxe, que le Tasse ne se soit pas mieux expliqué. Pour moi, je m'attache à ce que dit un Auteur ; et je ne sais pas lui faire dire ce qu'il ne dit point ${ }^{27}$.

La première citation est prise de la Jérusalem délivrée $\mathrm{du}$ Tasse $^{28}$ :

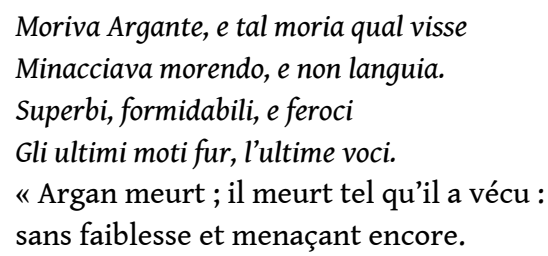


Superbes, formidables, féroces,

sont ses derniers moments et ses dernières paroles ${ }^{29}$ ".

La pensée prend cette forme dans la Jérusalem conquise ${ }^{30}$, texte moins connu en France :

Moriva Argante, e tal moria qual visse:

L'alma fuggia di Pluto al nero speco ;

Ma nella morte, e spaventosa faccia

Più terribil la morte ancor minaccia.

«Argan meurt; il meurt tel qu'il a vécu:

L'âme fuit jusqu'à l'antre noir de Pluton

Mais dans la mort, sa face effrayante

Plus terrible la mort encore menace. »

17 On pourrait presque traduire la citation en se servant des vers de la Thébaïde de Racine :

Et son âme en courroux s'enfuit dans les enfers.

Son visage, où la mort a répandu ses traits,

Demeure plus terrible et plus fier que jamais.

Ces derniers vers du Tasse étaient d'ailleurs autrefois signalés comme une source (par exemple dans l'éd. J.-L. Geoffroy de Racine parue en 1808). Aujourd'hui les notes ne donnent plus que Virgile...

Bouhours revient plus loin, dans le troisième dialogue, au motif du mort encore vivant. Il commence par condamner les pointes construites sur l'opposition entre la vie et la mort :

Mais pour ne point sortir de notre sujet, je mets au nombre des pensées qui pèchent par trop d'agrément toutes les antithèses recherchées, comme celle de vie et de mort, d'eau et de feu, dans les endroits que j'ai remarqués ${ }^{31}$.

20 Les pensées tournant sur des antithèses de l'eau et du feu sont bien connues et fréquentes dans le langage amoureux du xvII ${ }^{e}$ siècle. Ce sont celles dont nous disons aujourd'hui qu'elles sont pétrarquistes, même si, en fait, aux yeux d'un classique, elles sont plautiniennes ${ }^{32}$. Aux secondes appartiennent les pointes citées de Racine. Pour illustrer ces dernières, Bouhours donne cet exemple :

Florus, en parlant de ces braves soldats Romains qu'on trouva morts sur leurs ennemis après la bataille de Tarente avec l'épée encore à la main, et je ne sais quel air menaçant ; dit que la colère qui les animait lors qu'ils combattaient, vivait dans la mort même. Et in ipsa morte ira vivebat. C'était assez d'avoir dit qu'il restait sur leur visage un air menaçant: in vultibus minae. Il fallait s'en tenir là : et Tite-Live n'aurait eu garde de faire vivre la fureur guerrière dans la mort même ${ }^{33}$.

21 On pourrait dire la même chose des vers de Racine : c'était assez d'avoir dit qu'il restait sur son visage un air de colère. Il fallait s'en tenir là : et Tite-Live n'aurait eu garde de faire vivre la fureur guerrière dans la mort même.

\section{Les pointes dans La Thébaïde}

22 Les pointes de la fin du récit de Créon ne sont pas isolées. La pièce commence et finit par des pointes.

23 Le début de la pièce fonctionne sur un double registre. Le premier registre est celui de la parole adressée, il est très prosaïque :

Ils sont sortis, Olympe ? [...] Mais en sont-ils aux mains ? [...] N'en doutons plus, Olympe, ils se vont égorger. / Que l'on court avertir et hâter la Princesse ; / Je l'attends [...]. 
(l'Attila de Corneille, pièce créée aussi par la troupe de Molière, trois ans plus tard. Mais ce registre alterne avec un autre, celui de la parole non adressée à la suivante, parole entièrement faite de pointes. Une sortie des Thébains s'est faite grâce à Morphée qui avait endormi les assiégeants. Jocaste dormait encore. Elle se réveille, trop tard :

Qu'un moment de repos me va coûter de pleurs [...].

Mais l'art de la pointe culmine avec une adresse au soleil :

Ô toi, Soleil, ô toi qui rends le jour au monde,

Que ne l'as-tu laissé dans une nuit profonde !

À de si noirs forfaits prêtes-tu tes rayons,

Et peux-tu sans horreur voir ce que nous voyons?

Mais ces monstres, hélas ! ne t'épouvantent guères:

La race de Laïus les a rendus vulgaires,

Tu peux voir sans frayeur les crimes de mes fils,

Après ceux que le père et la mère ont commis.

Tu ne t'étonnes pas si mes fils sont perfides,

S'ils sont tous méchants, et s'ils sont parricides:

Tu sais qu'ils sont sortis d'un sang incestueux,

Et tu t'étonnerais s'ils étaient vertueux. [I, 1, éd. 1697].

Le passage participe de l'exposition et de la vraisemblance. Il indique qu'on est au petit matin juste après la sortie tentée par les Thébains et sert à démontrer au spectateur que la haine des deux frères est crédible. C'est extraordinaire, monstrueux, mais crédible et même banal, puisque c'est dans le mythe des Labdacides :

La race de Laïus les [= les monstres] a rendus vulgaires [...].

Mais le passage, tout en rappelant le vraisemblable de l'extraordinaire, convertit aussi le plus ordinaire en miracle: on s'y étonne que le miracle du soleil qui inverse son cours n'ait pas lieu! Bouhours comme hagiographe a suscité la haine de Voltaire. Dans sa biographie de François-Xavier, Bouhours signale que le saint a ressuscité huit morts. Voltaire se demande comment on peut croire des choses pareilles au siècle des Lumières. Cependant, ce n'est pas cela que Bouhours présente comme extraordinaire :

Le plus grand miracle du Xavier n'était pas d'avoir ressuscité tant de morts, mais de n'être pas mort lui-même de fatigue ${ }^{34}$.

De même, chez Racine, le plus grand miracle n'est pas que deux frères se détestent avant même la naissance, mais que le soleil se lève le matin.

L'invocation au Soleil de Jocaste est intéressante aussi par sa structure: un étonnement, suivi d'une explication. Cette structure rappelle les figures par ponderaçion mystérieuse, telles que les définit Gracián ${ }^{35}$ :

L'artifice de cette figure consiste à dresser une interrogation, un mystère dans la connexion des extrêmes entre les termes corrélats du sujet, c'est-à-dire entre ses causes, ses effets, adjoints, circonstances ou contingences [= différents « lieux» de l'« invention »]. Après avoir montré cette coöncidence et la question qu'elle pose, on en donne une solution, une réponse adéquate qui y satisfasse, expliquant le mystère. Remarquant que l'on faisait au soleil un inhabituel sacrifice, d'un cheval, Ovide en donna une explication par une sentence : «Ne celeri fieret victima tarda Deo ", au dieu de la célérité, on ne peut offrir d'animal paresseux ou lent. L'agudeza est dans le fait de ponderar [= peser ou donner du poids] l'étrange sacrifice, un cheval et non un éléphant ou un taureau et d'en donner ensuite une explication plausible [=équivalent $\mathrm{du}$ français plausible au sens classique, «ce qui mérite des applaudissements, de l'approbation », selon les termes du Dictionnaire de Furetière]. 
[...] Communément, la pondération éveille la curiosité, préparant le terrain à l'explication :

$$
\begin{aligned}
& \text { "C'est pourquoi au fils de Vénus } \\
& \text { On le peint tout nu et enfant } \\
& \text { Car tous les enfants ne savent } \\
& \text { Demander qu'en gémissant [...]. » }
\end{aligned}
$$

Le court poème cité par Gracián est de Góngora, mais c'est la réécriture d'un poème de l'Anthologie grecque :

Vois comme Amour ailé brise la foudre ailée, montrant par là qu'il est un feu plus fort que le feu. Ce n'est pas sans raison qu'Amour tient en main le dauphin et la fleur : puisqu'il possède à la fois la terre et la mer ${ }^{36}$.

C'est une épigramme tirée de l'Anthologie Planude, connue tôt, et une épigramme célèbre. La pensée ingénieuse qu'elle contient a fourni un sujet d'écriture dont Quintilien ${ }^{37}$ dit qu'il est usé à force d'avoir été proposé dans les écoles de rhétorique :

Quid ita crederetur Cupido puer, nudus, alatus, cum sagittis armatus?

L'épigramme a été reprise par Alciat dans ses emblèmes ${ }^{38}$.

Les derniers mots de la pièce de Racine sont aussi des pointes :

Ah! c'est m'assassiner que me sauver la vie

[...]

Et je m'en vais chercher du repos aux Enfers.

Le tout dernier vers fait écho, par la reprise du mot repos, à une des pointes du début :

Qu'un moment de repos me va coûter de pleurs.

Il se fonde sur un paradoxe simple : trouver du repos dans le lieu des supplices éternels. Sa vraie beauté est qu'il fait boucle avec l'ouverture de la pièce. On commence la pièce en ouvrant les yeux avec Jocaste, on la clôt en les fermant avec Créon. On commence en sortant du sommeil à la fin de la nuit, on finit en entrant dans la nuit de la mort. Le rapprochement entre le début et la fin se fonde sur une analogie, celle du sommeil et de la mort, analogie banale (somnum imago mortis, consanguineus Leti sopor, voir Homère et Virgile), et comme telle parodiée : dans une pièce de Rotrou, je crois, on dit à propos de vieilles femmes qui dorment en ronflant que jamais le sommeil n'avait été moins une image de la mort. En outre, Jocaste entre comme le fera Phèdre, en saluant le soleil. L'apparition de l'actrice s'accompagne ingénieusement d'une évocation de la lumière, possibilité du visible : les acteurs savent bien cela, qui font d'instinct une apostrophe à la lune ou au soleil en regardant un projecteur. La pièce est ainsi donnée comme un temps de visibilité, entre deux ténèbres (peut-être les ténèbres de la vraie vie).

On ne peut citer toutes les pointes de la pièce. Relevons cependant qu'elles peuvent organiser aussi le dialogue et les reparties :

JOCASTE :

«Il est aimé de tous. »

POLYNICE :

«C'est un tyran qu'on aime,

[...]

Et son orgueil le rend, par un effet contraire,

Esclave de son peuple et tyran de son frère. » (II, 3)

Il s'agit ici dans l'analyse de Gracián d'une figure par improportion tirée de la variété des adjoints (en espagnol adjuntos) ${ }^{39}$. Gracián donne un exemple tiré de Florus parlant des Romains voulant faire revenir Tarquin : 
Ils semblent vouloir que le peuple romain, qui est maître à l'extérieur, soit esclave à

l'intérieur de Rome ${ }^{40}$.

L'exemple est proche de la citation de Racine: ce sont deux pensées ingénieuses de blâme. La pensée y est tirée comme le précise Gracián de la variété des lieux, chez Racine elle est tirée de la variété des personnes.

L'accusation de Polynice dans les vers cités explicite un procédé du paradoxe, l'« effet contraire », un enchaînement par lequel se produit l'inverse de ce qu'on attend. Cette torsion logique revient souvent dans la pièce :

Le sang qui vous unit aux deux princes mes fils

Vous fait trouver en eux vos plus grands ennemis ; [I, 5]

Aussi bien mes respects redoublent vos mépris ; [I, 5]

Ses superbes discours tourneront à sa honte. [I, 6]

Serai-je parricide afin d'être bon père ? [III, 4]

Et je l'y mis, Attale, afin de l'en chasser. [III, 6]

Ils s'étouffent, Attale, en voulant s'embrasser. [III, 6]

Et je crains son courroux moins que son amitié. (IV, 1]

Par l'excès de leur haine il semblait réunis ; [V, 3]

Quelques-uns de ces effets contraires sont des maximes:

Plus l'offenseur m'est cher, plus je ressens l'injure. [I, 5 ; cf. Aristote, livre II de la

Rhétorique]

Une extrême justice est souvent une injure. [IV, 3 ; cf. summum jus summa injuria,

adage latin ${ }^{41}$.]

La loi de l'effet contraire semble parfois ordonner aussi la progression du discours. Un des moments de culmination de la pointe est celui des stances d'Antigone $(V, 1)$. Cellesci se donnent d'abord pour une délibération (« À quoi te résous-tu, princesse infortunée?»), mais aboutissent à une déclaration d'amour («Si jamais tu doutas de ma flamme fidèle...»). Elles fonctionnent donc comme une épigramme, où le début prépare la fin, mais en amenant cette fin par un « effet contraire » à ce qu'on attendait.

Les pièces suivantes de Racine contiennent moins de pointes que La Thébaïde. Il n'est pas sûr qu'elles contiennent moins de "pensées ingénieuses ». À l'annonce du meurtre de Britannicus, Burrhus a cette réflexion paradoxale :

Son crime seul n'est pas ce qui me désespère ;

Sa jalousie a pu l'armer contre son frère ;

Mais s'il vous faut, madame, expliquer ma douleur,

Néron l'a vu mourir sans changer de couleur.

[Britannicus, V, scène dernière, v. 1727-1730/v. 1708-1710]

Le propos ne s'accompagne pas de figures voyantes. Les figures ne se trouvent que dans un seul des trois vers : personnification discrète de la jalousie et métaphore lexicalisée armer contre. Pourtant il s'agit typiquement d'une " pensée ingénieuse ». Elle entre dans un type que Gracián appelle la «critique judicieuse », elle marque la prudence et la finesse de Burrhus :

Lorsque le commun commente un grand malheur, un échec manifeste, trouver et en démontrer un autre encore plus grand, mais moins évident, est grande affaire de cette judicieuse subtilité. Ainsi, le grand duc d'Albe ne condamnait pas tant Pompée pour avoir été vaincu de ses ennemis que pour s'être laissé convaincre par ses amis, alors qu'il était d'un avis contraire, de livrer à César une bataille qui se devait avérer funeste ${ }^{42}$. 


\section{De la pensée ingénieuse à l'ingéniosité du scénario}

[...]

\section{Un vers comme} coup de théâtre (peripeteia).

On remarque que bien des pointes de La Thébaïde peuvent être lues comme un commentaire que la tragédie livre sur elle-même. La plainte de Jocaste à la scène 4 de l'acte III est aussi une définition de la péripétie, au sens moderne du terme :

Connaissez mieux du ciel la vengeance fatale :

Toujours à ma douleur il met quelque intervalle,

Mais hélas! quand sa main semble me secourir,

C'est alors qu'il s'apprête à me faire périr.

Il feint de s'apaiser, et devient plus sévère :

Il n'interrompt ses cours que pour les redoubler,

Et retire son bras pour me mieux accabler.

Ce trône fut toujours un dangereux abîme [IV , 3]

formule de façon dense et frappante un trait qui définit traditionnellement la tragédie, le renversement qui menace les grands de les faire passer du bonheur au malheur. Il en est de même de ce regret, autrement exprimé au début de l'Iphigénie (v. 10-12) :

Et fera regretter aux princes des Thébains

De n'être pas sortis du dernier des humains. [II, 1]

L'« effet contraire » a d'ailleurs un correspondant dans la Poétique d'Aristote : c'est le

Le coup de théâtre est, comme on dit, le renversement qui inverse l'effet des actions, et ce, suivant la formule, vraisemblablement ou nécessairement. Par

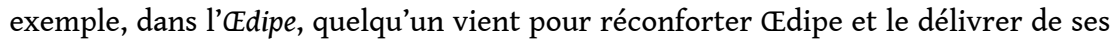
craintes au sujet de sa mère ; mais, en lui révélant son identité, il fait l'inverse. Dans le Lyncée, on conduit un personnage à la mort tandis qu'un autre, Danaos, l'accompagne pour le tuer ; mais ce qui résulte du déroulement des actions c'est que Danaos meurt tandis que l'autre est sauvét3.

La torsion logique du coup de théâtre n'est pas chez Aristote définitoire de la tragédie. Des reformulations telles que celle de Chapelain semblent pourtant le suggérer :

La poésie représentative, aussi bien que la narrative, a pour objet l'imitation des actions humaines, pour condition nécessaire la vraisemblance, et pour perfection la merveille. De l'artificiel assemblement du vraisemblable et du merveilleux naît la dernière beauté des ouvrages de ce genre ; et ces deux parties sont de l'invention ${ }^{44}$.

C'est que la Poétique d'Aristote a été lue au XvII siècle comme un traité pour obtenir la «merveille ». Il est vrai que pour susciter la terreur et la pitié il faut selon Aristote bien des paradoxismes: prendre pour sujet un conflit non entre ennemis, mais entre proches, entre parents ; mettre en scène des héros à la fois bons et méchants. Le soustitre de la pièce de Racine, Les frères ennemis, dit la conformité de son sujet avec le sujet tragique selon Aristote, mais c'est d'abord un oxymore.

Les travaux de Georges Forestier ont montré que Corneille avait théorisé un processus d'écriture de la tragédie "à rebours ", en commençant par la fin ${ }^{45}$. C'est en somme écrire une tragédie comme on fait une épigramme. Guillaume Colletet dans son traité sur l'épigramme écrit : « selon la maxime des Philosophes, la fin doit être la première dans l'intention, et la dernière dans l'exécution ». Il cite l'adage Respice finem, qui vaut pour conseiller la prudence, mais qui est aussi «le grand secret » du Poète ingénieux ${ }^{46}$. 

aspects de la création racinienne. Créon devrait être l'ennemi d'Antigone ; il en devient l'amant, contre les sources. La relation entre Créon et Antigone peut ainsi redoubler l'amour monstrueux entre Jocaste et ๔dipe. Ces couples entrent eux-mêmes en relation inverse avec celui des «frères ennemis ». Est ainsi créé un cristal qui enchanterait un anthropologue structural : sur-évaluation et sous-évaluation des relations de sang. La pièce raconte la haine de ceux qui sont très proches, mais aussi l'amour de ceux qui devraient rester éloignés. La loi de composition est explicitée dans la pièce, quoiqu'attribuée par Étéocle au Ciel et non bien sûr au dramaturge :

Et que dans notre sang il voulut mettre au jour

Tout ce qu'ont de plus noir et la haine et l'amour. [IV, 1]

Le traitement du personnage de Créon par redoublement d'un paradoxe est donc, si l'on y réfléchit, exactement celui d'Hippolyte dans la dernière pièce de Racine pour la scène publique, treize ans plus tard. On sait que dans Phèdre, Racine viole sa source mythologique : l'histoire n'est plus du tout celle de la vengeance de la déesse de l'amour bafouée par un "garçon insensible». Hyppolyte devient un fils d'amazone tendre, c'est-à-dire un personnage oxymorique, faisant paire avec un autre personnage oxymorique, Phèdre peinte en marâtre aimante. La fortune malheureuse de La Thébaïde ne vient pas d'une mauvaise construction du personnage de Créon, mais bien plutôt du fait qu'après la philosophie allemande du $\mathrm{xIx}^{\mathrm{e}}$ siècle le conflit entre Créon et Antigone est devenu inoubliable ${ }^{47}$. À cause d'elle, nous ne nous sommes pas prêts à oublier l' Antigone de Sophocle, alors même que nous oublions Euripide en lisant la Phèdre de Racine. C'est à cause de Hegel que nous trouvons mauvaise la première pièce de Racine, comme c'est à cause de Freud que nous ne trouvons plus intéressant l'œdipe de Corneille.

Mithridate est une pièce entièrement fondée sur la pensée ingénieuse. La pièce réunit trois éléments séparés dans les textes historiques : la défaite de Mithridate, le rapport de Mithridate et de ses fils, le mariage avec Monime. Racine dit avoir choisi Monime plutôt qu'une autre femme, car ce fut «la plus vertueuse, celle qui l'a aimé le plus tendrement». Mais elle est surtout celle qui fournit une belle pointe tragique, puisqu'elle utilise le tissu de son mariage comme instrument de sa mort : «Ô maudit et malheureux tissu, ne me serviras-tu point au moins à ce triste service? » La pointe renouvelle celle, commune, du tombeau où, par un « effet contraire », entre celle qui devait entrer dans le lit nuptial. La même pointe se retrouve dans Bajazet.

Le sujet de la pièce est la mort de Mithridate : c'est ce que nous suggère le principe de construction « à rebours » (puisque c'est le dénouement) et c'est ce que dit Racine («sa mort qui est l'action de ma tragédie »). Ce sujet a déjà été traité. Racine le renouvelle grâce à l'« effet contraire » : ce n'est pas la défaite qui entraîne la mort de Mithridate, comme dans la pièce pathétique de La Calprenède par exemple, c'est son projet de conquête qui est «en partie cause de sa mort qui est l'action de ma tragédie ». Or ce projet est bien sûr une merveille. Comme d'autres pièces nous montrent un heureux naufrage, une heureuse ingratitude, un hypocondriaque amoureux, ou un bourgeois gentilhomme, Mithridate nous montre une glorieuse défaite. Mithridate devient le « nom » même de la glorieuse défaite, comme le dit le début de la préface. La pièce est comme le «corps" d'une devise dont l'«âme» pourrait être, pour reprendre des «âmes» de devise commentées par Bouhours lui-même dans les Entretiens d'Ariste ${ }^{48}$, quelque chose comme clarior adversis (plus brillant dans l'adversité) ou plutôt 
major ab adversis (ce qui s'oppose à lui nous le fait voir plus grand), car, comme le dit Racine lui-même dans sa préface : "ses seules défaites ont fait presque toute la gloire de trois des plus grands capitaines de la République [romaine]». De ce motif de la glorieuse défaite, Bouhours ne manque pas de parler, et il l'associe à celui du personnage tué et encore vivant :

Je ne vous en dis plus qu'une [= une pensée du Tasse] que je ne puis me dispenser de vous dire, tant le raffinement y est visible : c'est à l'occasion du combat de Tancrède et de Clorinde. II dit que les deux combattants se font l'un à l'autre avec leurs épées des plaies profondes et mortelles; et que si l'âme ne sort point par de si larges ouvertures, c'est que la fureur les retient:

$$
\begin{gathered}
\text { Ese la vita } \\
\text { Non esce, sdegno tien la al petto unita }{ }^{49} \text {. }
\end{gathered}
$$

II a, repartit Philanthe, une pensée toute contraire, en parlant d'un Sarasin qui combattit vaillamment jusques au dernier soupir, et qui fut si couvert de blessures, que son corps parut n'être qu'une plaie.

E fatto è il corpo suo solo una piaga ${ }^{50}$.

Car il dit ensuite: Ce n'est pas la vie, c'est la valeur qui soutient ce cadavre indomptable, et furieux dans le combat.

$$
\begin{aligned}
& \text { La vita nò, mà la virtù sostenta } \\
& \text { Quel cadavero indomito, e feroce }{ }^{51} \text {. }
\end{aligned}
$$

Tout cela, répondit Eudoxe, me paraît trop fin et trop recherché.

Que direz-vous donc, répliqua Philanthe, de ce qu'on a écrit sur ce brave Grec qui mourut debout, tout percé de flèches à la bataille de Marathon, et qui se tint droit après sa mort, soutenu des flèches qui le perçaient de toutes parts? Vous voulez parler, dit Eudoxe, de la Harangue qu'un docte Hollandais [en marge: Daniel Heinsius] fait faire par forme de Déclamation au père de Callimaque, et qui est a la fin des deux Éloges funèbres de Cynégire et de Callimaque, qu'un savant Jésuite [en marge : Petris Possinus] a traduits en Latin du Grec de Polémon le Sophiste ? Cette Harangue est pleine de traits assez vifs; mais il m'y parait une affectation exquise depuis le commencement jusqu'à la fin : je l'ai relue depuis quelques jours, et j'ai marqué les endroits qui brillent le plus; je vais vous les lire.

Il y a lieu de douter, c'est le père de Callimaque qui parle, si mon fils a vaincu en mourant, ou est mort en vainquant. La mort n'a point interrompu sa victoire, mais elle l'a continuée. Il a soutenu toute l'Asie et n'est point tombé. Il est mort, et est demeuré debout. Nature, pourquoi lui avez-vous donné un esprit céleste, ou un corps mortel ? Il n'a pu ni tomber, ni être vaincu, et il a été contraint de mourir. Il n'a pas quitté son corps, mais son corps l'a quitté. Il est le premier qui a cédé à la nature en triomphant d'elle. Il est le premier que la mort n'a point abattu, qui a donné après son trépas des marques de sa valeur, qui a étendu, par la mort même, la gloire et la durée de sa vie. Je ne sais si je dois demander pour lui, ou refuser un mausolée. Plût à Dieu, Callimaque, que tu pusses parler après ta mort, comme tu as pu vaincre! Tu répondrais sans doute en ces termes: [...]. La postérité n'aura pas plus de peine à croire qu'un mort ait combattu, qu'à croire qu'il ne soit pas tombés2. cependant tout du long. Et dans les Pensées ingénieuses, il donne un recueil de pensées pris à Tite-Live, dont celle-ci :

Inter tantas fortunae minas metuandas magis quam metuens. [lib. 2]

«Un homme fier et courageux se fait quelquefois plus craindre dans la disgrâce qu'il ne craint lui-même ${ }^{53}$.»

Pour conclure ces remarques sur Racine et Bouhours, trois propositions. 

peut-être travailler sur les rapports entre la rhétorique de Racine et l'enseignement jésuite. Je ne donne cette première proposition que pour rire : la " mise en intrigue " actuelle des études raciniennes ne laisse pas espérer ces travaux pour bientôt. Et pourtant : la rhétorique de Bouhours est anti-janséniste ; or l'esthétique de Racine est proche de celle de Bouhours; ergo. largement la notion de style au XVII ${ }^{\mathrm{e}}$ siècle. On a souvent considéré qu'il y avait un «style de Racine », mais que celui-ci n'était pas encore au point dans La Thébaïde, où il était encore alambiqué. On avance souvent l'idée d'un jeune Racine encore précieux, sans expliquer la révolution qui fait passer d'un style de jeunesse présent en 1664 à un style classique à partir de 1665 . Georges Forestier réfute cette idée et propose d'y voir un style qui reflète dans l'élocution les jeux d'antithèses au cœur même de l'invention ${ }^{54}$. Mais d'autres pièces jouent sur les oppositions au niveau de l'invention et n'ont pas ce style alambiqué. Le rapprochement entre la tête de Pompée et le visage d'Étéocle au cœur des récits de La Mort de Pompée et de La Thébaïde suggère une hypothèse. Corneille avec La Mort de Pompée revendique avoir réussi à faire une tragédie dans le style de Lucain, comme il avait essayé, mais sans y arriver parfaitement, d'écrire Médée dans le style de Sénèque ${ }^{55}$ :

Pour le style, il est plus élevé en ce poème qu'en aucun autre des miens; [...] j'ai traduit de Lucain tout ce que j'y ai trouvé de propre à mon sujet, et [...] j'ai tâché pour le reste à entrer si bien dans sa manière de former les pensées et de s'expliquer que ce qu'il m'a fallu joindre du mien sentît son génie, et ne fût pas indigne d'être pris pour un larcin que je lui eusse fait ${ }^{56}$.

Dans une préface publiée douze ans après la première édition de la pièce, Racine évoque certes Euripide comme source pour le plan de sa tragédie, mais on pourrait supposer que pour le style, il aurait visé « la manière de former les pensées » propre à Stace. Le titre choisi pour la pièce, La Thébaïde, est d'ailleurs celui-là même de l'épopée de ce dernier, ce qui risque de passer aussi inaperçu que la fameuse lettre volée de Poe. Si le tragique est dans cette pièce conçu comme " héroïque ", il n'est plus alors étonnant d'y trouver des passages pris à l'Énéide ou au Tasse, plutôt qu'à Euripide. Il n'est pas étonnant non plus que le style nous semble alambiqué. Les érudits actuels notent le «baroquisme» du style de Stace. Le recueil de Jugements des savants sur les principaux ouvrages... d'Adrien Baillet complété par de la Monnoye nous donne une idée des jugements classiques sur Stace ${ }^{57}$. Celui-ci est mis très haut et incarne le style véhément. En même temps, son style est soupçonné d'enflure ou d'affectation. Scaliger prétend qu'aucun auteur antique, Homère compris, n'a approché Virgile comme l'a fait Stace. Le jugement sera repris par le traducteur Marolles en plein classicisme. Scaliger note toutefois que "naturellement élevé, il n'a pu éviter de devenir enflé et trop bouffant ${ }^{58}$ ». Le Père Rapin trouve qu'il est «aussi bizarre dans ses idées que dans ses expressions ». Son style varie un peu d'œuvre en œuvre et, dans sa Thébaide, « il est plus peigné, plus ajusté et plus fardé ${ }^{59} »$. Stace incarne le style violent héroïque, celui qui s'oppose au style doux lyrique. Racan selon Ménage aurait témoigné de l'amour de Malherbe pour Stace. Ceux qui veulent réfuter cette admiration au profit du seul Horace décrivent ainsi l'opposition entre le style de Malherbe et celui du Stace :

Le caractère de Malherbe [...] est éloigné de celui de Stace autant que le Ciel l'est de la Terre. [...] L'un est le Poète Lyrique, l'autre est le Poète Héroïque, l'un joue du luth, l'autre bat du tambour. Malherbe est doux et réglé ; l'autre est emporté et 
violent. [...] Ce n'est pas que Stace n'ait ses charmes, mais ses beautés et celles de Malherbe sont toutes différentes, car on voit en celui-ci un visage serein, et une Majesté douce et tranquille ; au lieu que Stace n'a rien que de terrible, et qu'il a mis tous ses charmes dans un certain air belliqueux et plein de fiertéteo.

Cette théorisation de l'invention proprement poétique gagnerait à mon avis à faire se croiser la poétique dramatique et la poétique de l'ingéniosité fondée sur la tradition de l'épigramme comme forme matricielle de la littérature, à faire se croiser la théorie de la dramaturgie classique et celle de la «manière de bien penser dans les ouvrages de l'esprit ». À cette condition, la rhétorique pourrait peut-être vraiment éclairer la tragédie racinienne. ressemblent de façon troublante aux reproches faits à la Thébaïde de Racine ${ }^{61}$.

C'est la troisième et dernière proposition sur laquelle je voudrais mettre l'accent, car elle concerne l'objet de l'Équipe RARE, la rhétorique, et le sujet même de ce numéro, la question du rapport entre rhétorique et tragédie racinienne.

Dans les études qui concernent cette question, on ne sait pas trop quoi faire de la rhétorique épidictique. Une première solution consiste à réserver la question à un autre jour et on ne parle que de la rhétorique délibérative et de la rhétorique judiciaire. Dans la tragédie pourtant, les situations délibératives et judiciaires sont le plus souvent " truquées ", c'est-à-dire irrégulières. Souvent la tragédie met en scène la délibération et le jugement comme des leurres et elle met plus souvent en scène l'échec que le triomphe de la rhétorique « intra-scénique » (selon la terminologie de Gilles Declercq). La clémence d'Auguste au dénouement de Cinna n'est merveilleuse que parce qu'Auguste n'a pas été convaincu par le discours de clémence de Livie à l'acte précédent. Bien plus, un discours aboutit souvent, par un « effet contraire ", à l'inverse de ce qu'il voulait atteindre: le faux triomphe du parfait discours d'Agrippine à l'acte IV de Britannicus en est un bon exemple. La merveille n'est pas dans le fait d'offrir des morceaux de rhétorique, mais de les insérer ingénieusement dans des situations.

Une seconde solution est de réduire l'épidictique à des détails présents dans des discours d'autres genres, par exemple à une congratulatio d'un discours exhortatif, ou à la captatio amicitiae d'un discours judiciaire. C'est peu.

Une dernière solution, radicale, mais peu recommandable, est de redéfinir à sa convenance ce qu'est l'épidictique.

Cette mise à l'écart de l'épidictique est d'autant plus gênante que toute une tradition associe la poésie et particulièrement la tragédie à l'épidictique. Corneille rappelle que cette tradition s'appuie sur la Poétique d'Aristote lue par Averroès ${ }^{62}$. La place de l'épidictique est peut-être à chercher non dans la rhétorique "intra-scénique ", mais au cœur même de l'invention, l'invention qui est dans la théorie classique ce qui distingue fondamentalement rhétorique et poétique, et qui, dans la tradition aristotélicienne, s'assimile à la seule tâche propre au poète :

De l'artificiel assemblement du vraisemblable et du merveilleux naît la dernière beauté des ouvrages de ce genre ; et ces deux parties sont de l'invention ${ }^{63}$. 


\section{NOTES}

1. Nous utiliserons ici par commodité le mot de pointe, mais en France à l'époque classique il n'est employé que pour désigner les «pensées ingénieuses » burlesques ou, avec une valeur péjorative, les « pensées ingénieuses » qui tombent dans l'affectation et que pour cela on condamne.

2. Notice sur D. Bouhours dans G. Grente dir., Dictionnaire des lettres françaises, XVII ${ }^{e}$ siècle, éd. revue sous la dir. de P. Dandrey, Paris, Fayard, 1995, p. 193-195.

3. Lettre $n^{\circ} 45$ de l'éd. P. Mesnard des CEuvres de Racine, t. 6, Paris, Hachette, 1865, p. 515-516.

4. Le catalogue de la $\mathrm{BnF}$ répertorie seize éditions de 1680 à 1771 . Nous citons la réédition de 1743, Paris, Charles-Nicolas Poirion. Une reproduction de l'édition de 1705 a été donnée par S. Guellouz, Toulouse, Société de littératures classiques, 1989.

5. La Manière de bien penser, « Avertissement », op. cit., p. vj.

6. Vingt-quatre éditions entre 1671 et 1768.

7. Une édition critique en a été donnée par B. Beugnot et G. Declercq, Paris, Champion, 2003. On trouve dans cette édition une bibliographie (p. 541-566).

8. Nous citons l'édition de 1734, Paris, veuve Delaulme, reproduite par Sussex reprints, Brighton, 1971.

9. Sur cette tradition pédagogique, voir A. Moss, Printed Commonplace-Books and the Structuring of Renaissance Thought [1996], trad.fr. Les recueils des lieux communs: Apprendre à penser à la Renaissance, trad. de l'anglais par P. Eichel-Lojkine et alii, Genève, Droz, 2002.

10. La Manière de bien penser, "Avertissement », op. cit., p. vj.

11. Ibid., « Premier dialogue », p. 19.

12. M. Blanco, Les Rhétoriques de la Pointe. Baltasar Gracián et le Conceptisme en Europe, Genève, Slatkine, 1992.

13. Pensées ingénieuses, «Avertissement », op. cit., ã ij vº Cette classification se trouve déjà dans La Manière de bien penser.

14. B. Gracián, Art et figures de l'esprit. Agudeza y arte del ingenio (1647), trad. B. Pelegrín, Le Seuil, 1983, p. 268.

15. Voir sur le site de l'équipe RARE (Rhétorique de l'Antiquité à la Révolution), les loci dans les commentaires rhétoriques de Ferrazzi. URL: http://w3.u-grenoble3.fr/rare/spip/spip.php? article 167

16. Racine, Théâtre-Poésie, Paris, Gallimard, 1999, p. 1271 et La Thébaïde, Paris, Gallimard, 2010 (coll. « Folio/Théâtre »), p. 203.

17. Trad. M. Rat, Paris, Garnier, 1965, rééd. coll. GF-Flammarion.

18. Chant XLVI, 140, trad. F. Reynard, Paris, Gallimard (coll. « Folio classique »), 2003, t. II, p. 471.

19. C. Lanzmann, Le Lièvre de Patagonie, Paris, Gallimard, 2009, rééd. coll. « Folio », 2010, p. 37.

20. XI, v. 568-569, trad. R. Lesueur, Paris, les Belles Lettres, t. 3, 1994.

21. La Pharsale en vers français de Brébeuf, Paris, A. de Sommaville, éd. 1659, p. 311.

22. Corneille, Pompée, III, 1, v. 761-769 dans Euvres complètes, éd. G. Couton, Paris, Gallimard, t. I, p. 1103.

23. Op. cit., p. 408.

24. La Manière de bien penser, « Premier dialogue », ibid., p. 15-20.

25. Mme de Sévigné, Lettre du 8 mars 1676 dans Correspondance, éd. R. Duchêne, Paris, Gallimard, t. II, 1986, p. 249.

26. II, XXIV, 60.

27. La Manière de bien penser, " Premier dialogue », op. cit., p. 16-18.

28. Chant XIX, st. 26. 
29. Trad. A. Desplaces, 1858, reprint Les introuvables, 1976, p. 329. Argan est un Sarrasin tué par Tancrède.

30. XXIII, 105.

31. La Manière de bien penser, "Troisième dialogue ", op. cit., p. 362.

32. P. Laurens, L'Abeille dans l'ambre: célébration de l'épigramme de l'époque alexandrine à la fin de la Renaissance, Paris, les Belles lettres, 1989, p. 386-387.

33. Le texte est tiré de l'Epitome rerum romanorum (I, XVII): Omnium vulnera in pectore, quidam hostibus suis immortui, omnium in manibus enses, et relictae in vultibus minae, et in ipsa morte ira vivebat. [Tous étaient blessés à la poitrine : quelques-uns étaient morts sur les cadavres de leurs ennemis : l'épée était dans leurs mains, la menace sur leurs fronts, et, dans la mort même, leur courroux vivait encore.]

34. Voltaire, Dictionnaire philosophique, article « François Xavier».

35. B. Gracián, Art et figures de l'esprit, Discours VI, op. cit., p. 114-115.

36. Poème traduit et cité par P. Laurens, op. cit., p. 436-437.

37. Institution oratoire, XI, 4, 26.

38. Emblème 113, cité par P. Laurens, op. cit., p. 439.

39. Sur les adjoints comme un des «lieux» de l'« invention », voir sur le site RARE, les adjuncta dans les commentaires rhétoriques de Ferrazzi. URL : http://w3.u-grenoble3.fr/rare/spip/ spip.php?article167

40. B. Gracián, op. cit., Discours V, p. 110.

41. L'adage est transmis par Cicéron (Des Devoirs). La même pensée ingénieuse sert à un "mot " d'Arletty (qui fait allusion à l'«épuration» après 1944). On lui dit: "Quoi de pire que l'injustice? » Elle répond : « La justice. »

42. B. Gracián, op. cit., p. 201-203.

43. Début du chapitre 11, éd. R. Dupont-Roc et J. Lallot, Paris, Le Seuil, 1980, p. 71.

44. Chapelain, «Discours de la poésie représentative" dans Opuscules critiques, éd. A. C. Hunter, revue par A. Duprat, Genève, Droz, 2007, p. 272.

45. Sur la notion de construction "à rebours", voir G. Forestier, Essai de génétique théâtrale. Corneille à l'œuvre, Paris, Klincksieck, 1996 (en particulier p. 127-144).

46. G. Colletet, L'Art poétique, "Traité de l'épigramme»,1658, section XV, éd. P.- J. Jannini, Genève, Droz et Paris, Minard, 1965, p. 83.

47. On sait que Hegel fit de la lutte de Créon et d'Antigone le modèle même du tragique, comme illustrant le conflit de la «rationalité objective de l'État» et de l'«amour sacré pour le frère », «force tout aussi morale", conflit montrant que "l'idéal en se déterminant s'oppose à luimême" (Esthétique). Antigone a eu aussi l'honneur d'une traduction par Hölderlin (1804), d'analyses philologiques d'inspiration hégélienne (August Boeckh, 1824) et de représentations « à l'antique » en 1841 à la cour du roi de Prusse à Postdam, puis dans différentes villes européennes avec une musique de Mendelssohn. Voir P. Demont et A. Lebeau, Introduction au théâtre grec antique, Paris, LGF, 1996, p. 223-226. Plus largement, sur l'invention du tragique par l'idéalisme allemand, voir P.Szondi, Versuch über das Tragische,1961,trad.fr. Essai sur le tragique, Paris, Circé, 2003.

48. Op. cit., p. 449 et 447.

49. Jérusalem, chant XII, 62, trad. citée, p. 225 : «Oh! Qu'elles sont larges et sanglantes, les ouvertures que font leurs épées dans les cuirasses et dans les chairs! Si la vie ne s'échappe pas de leurs poitrines, ce n'est que la fureur qui les retient. »

50. Ibid., chant VIII, 22, p. 156 : «Tout son corps n'est qu'une seule plaie. »

51. Ibid., chant VIII, 23, p. 156: «Ce n'est plus la vie, mais le courage qui soutient ce corps indompté. »

52. La Manière de bien penser, « Troisième dialogue ", op. cit., p. 393-394.

53. Pensées ingénieuses, op. cit., p. 709 sq. 
54. Racine, La Thébaïde, coll. « Folio-théâtre »,p. 23 (" Et c'est comme naturellement que la figure rhétorique fondamentale qui traverse les discours de tous les personnages et qui transcende toutes les oppositions soit la figure de l'antithèse »).

55. Voir les derniers paragraphes des «Examens » de Médée et de La Mort de Pompée.

56. Corneille, CEuvres complètes, op. cit., t. I, p. 1077.

57. T. 3, Seconde Partie, Poètes latins, $n^{\circ}$ 1166, p. 347-355.

58. Ibid., p. 348.

59. Ibid., p. 351.

60. Lettre de Mosan de Brieux cité par A. Baillet, Jugements, t. 4, seconde partie, 1725, p. 10.

61. A. Baillet, Jugements, t. 3, seconde partie, p. 354.

62. Corneille, Don Sanche d'Aragon, "Épître dédicatoire à M. de Zuylichem " dans CEuvres complètes, op. cit., t. II, p. 552.

63. Chapelain, op. cit., p. 272.

\section{AUTEUR}

JEAN-YVES VIALLETON

Univ. Grenoble-Alpes, RARE - Rhétorique de l'Antiquité à la Révolution 